\title{
CHEMICAL MODELS OF ACTIVE REGIONS
}

\author{
S.B. CHARNLEY ${ }^{\dagger}$ \\ Space Science Division, NASA Ames Research Center, California 94035.
}

\begin{abstract}
Theoretical models of the chemistry in regions of low-mass star formation are re-
\end{abstract} viewed.

\section{Introduction}

I will interpret active regions as those regions in which young, low-mass stars are forming. Other regions where dynamical processes directly influence the chemistry are described in the contributions by Walmsley, Prasad and Ziurys. The physical conditions pertaining to regions of low-mass star formation have been reviewed recently by Cernicharo [5].

Theoretical models of molecule formation in dense interstellar clouds have tended to concentrate on the chemistry and to neglect the physical evolution of the gas (Millar [23] and references therein). 'Pseudo-time-dependent' [21], or static, models assume that the density and temperature of the gas remain constant throughout the age of a cloud and neglect, apart for $\mathrm{H}_{2}$ formation, the gas-grain interaction. Under these assumptions a chemical steady-state is attained after about $10^{7}$ years, however, detailed comparison with observed molecular abundances show that the abundances at times $\sim 10^{5}$ years appear to be in better agreement. Ice mantles $\left(\mathrm{H}_{2} \mathrm{O}, \mathrm{CO}, \mathrm{CH}_{3} \mathrm{OH}\right)$ containing significant fractions of the available heavy elements are widely observed in molecular clouds [29]. The characteristic time for complete accretion of heavy gas phase species on to dust grains is typically $\sim 10^{6}$ years, and so, in the absence of a continous desorption mechanism [12], one must have a means of returning solid material to the gas phase. Static models that do allow for the formation of molecular ices, and do not return mantle material, show that a complete freeze-out occurs well before a steady-state is reached, and also that the chemical evolution at earlier times is different in a non-trivial way [4].

Molecular clouds are observed to be the sites of star formation and exhibit a complex, clumpy, structure with material co-existing in several density phases. The effect of star formation is to disrupt the ambient medium via the associated outflows and shocks. It has been suggested that the periodic removal of mantle material by processes associated with star formation, on a time-scale comparable with the local star formation rate, can return mantle material to the gas phase and maintain a large $\mathrm{C} / \mathrm{CO}$ ratio [30]. In the dark cloud Barnard 5 (B5) it has been shown that the outflows from embedded young stars are interacting with the surrounding clumpy medium from which they originally formed [16]. Estimates suggest that the gas density changes on a time-scale of about $6 \times 10^{5}$ years, shorter than the time-scales of accretion and chemical steady-state. The important conclusion is that the chemistry in dense interstellar clouds is of a nonequilibrium nature [16]. Chemical kinetic models of these regions should therefore attempt to include the gas-grain interaction, and also the density and temperature variations associated with collapse, expansion, †NAS/NRC Resident Research Associate 
and shocks [11]. In this review I briefly describe recent work on modelling the chemistry in starforming regions, with particular reference to B5.

\section{Theoretical Models}

A theory of the physics of clumpy molecular clouds regulated by star formation was developed by Norman \& Silk [26]. In the Norman-Silk model, gas and dust are continously cycled between dense $\left(n \sim 10^{4} \mathrm{~cm}^{-3}\right)$ clumps and the interclump medium $\left(n \sim 10^{3} \mathrm{~cm}^{-3}\right)$. The winds from young stars create bubbles and disrupt the surrounding medium. The chemistry in these wind-blown bubbles has been modelled [6] and included such processes as the ablation and entrainment of clump material by stellar winds, chemistry in the reverse shock at the bubble edge, and depletion on to grains as the cool shell is disrupted, fragments and reforms the next generation of clumps. The assumed presence of ionised wind material $\left(\mathrm{H}^{+}\right.$and $\left.\mathrm{He}^{+}\right)$in the mass-loaded wind [18] can limit the conversion of heavy atoms into hydrides in the post-shock gas. The high pre-shock fractional ionisation, $x_{e}\left(\sim 10^{-2}\right)$, means that the shock is J-type [13].

Two types of model have been developed specifically for the nonequilibrium chemistry in B5 [7]. In the first, based upon a scenario in which gas and dust is rapidly cycled between clump and interclump phases on a time-scale of $6 \times 10^{5}$ years [16], clumps form at the free-fall rate and the shocks which occur are C-type [13]. In a second model a much slower clump formation rate and longer lifetime was considered which led to a cycling time-scale of $6 \times 10^{6}$ years. Partial ionisation of stellar winds by strong bow shocks and entrainment of clump material into the winds was also considered. In both models the formation and removal (by shock waves) of molecular mantles was important. Calculations showed that the chemistry was sensitive to the adopted dynamics and that the models could produce repeatable abundances between cycles. In these studies the grain surface formation of saturated molecules had a significant effect on gas phase chemistry.

The chemistry of nitrogen-bearing molecules in star-forming clouds has been studied in the context of the slow cycling models described above [25]. The abundances of several molecules in cold clumps were found to be determined by the post-shock chemistry (e.g. HCN) and by formation on grains (e.g. $\mathrm{NH}_{3}$ ).

A simple model has been developed to study the chemistry which may occur at wind-clump interfaces as hot, ionised, wind material mixes with the cold gas at the surfaces of clumps embedded in the flow. Calculations indicated that it may be possible to elucidate chemical diagnostics of the interface physics. For example, when both mixing and heating occur with high efficiency the $\mathrm{CH}$ molecule is predicted to become very abundant in the interface region [8].

The formation and removal of ice mantles is a fundamental feature of the cycling models and they predict spatial and temporal variation in the mantle size throughout the cycle associated with a particular dynamical model. Ice mantles have been detected in B5 towards the infrared source IRS1 [9]. The time-scale required to form the observed column density of ice has been used to constrain the clump formation time-scale and hence also the dynamics of the region. Fitting of the $3 \mu \mathrm{m}$ feature has been used to infer the composition and thermal history of the ice mantles during a star formation cycle [10].

To test the validity of various aspects of the theoretical work outlined above, multi-molecular studies of star-forming regions are required. For the case of B5 such a study would be particularly useful as the chemistry in this cloud has been the focus of a substantial amount of theoretical work [11]. Such studies have been performed for other star-forming regions. In B1, significant differences in the abundances of methanol, $\mathrm{SiO}$ and complex hydrocarbons, compared to other clouds, have been found [1]. Observations of $\rho$ Oph [22] have illustrated the clumpy nature of this region and the stong spatial variation in the emission of several molecules. Recent maps of NGC 2071(N) also show dramatic spatial differences in molecular emission and it has been proposed that these arise from differences in molecular abundances due to the effects of local star formation [17]. 
It would be interesting to model the above sources in the future.

It has recently been suggested that the chemistry of TMC-1, long considered the typical cold, quiescent cloud, is the result of local dynamical interactions, due specifically to collapse of diffuse gas in Heiles Cloud 2 [31]. An alternative dynamical origin for TMC-1 has been proposed to account for its apparently peculiar chemistry : the cloud is a shock remnant [27]. Appreciable abundances of methane and acetylene can be produced in diffuse cloud shocks $[24,28]$ and provide the initial conditions for the synthesis of large hydrocarbons by ion-molecule reactions [19]. Models of TMC-1 in which diffuse gas is shocked, followed by collapse to the required density, can produce large abundances of cyanopolyynes [3].

\section{Discussion}

Barnard 5 has been observed in ${ }^{12} \mathrm{CO},{ }^{13} \mathrm{CO}, \mathrm{C}^{18} \mathrm{O}, \mathrm{CS}, \mathrm{NH}_{3}$ and $\mathrm{HCN}[2,15,16]$, as well as in ice, and so one may begin to compare the theoretical predictions. The observed $\mathrm{HCN}$ abundance of $2.8 \times 10^{-9}$ [15] in the core IRS1, taken with the observed ice column density in this line of sight [9], are particularly interesting as they place constraints on the published cycling models of nitrogen chemistry. These calculations have only been performed for the slow cycling models in which ablation and mixing are important. The HCN abundance in the cold clump phase reflects its value in the post-shock gas, as the clump ages gas phase HCN is lost by accretion on to grains. The minimum time, $t_{m i n}$, to form the observed ice mantles (roughly the minimum clump age) in each of the fast and slow cycling models is $7.6 \times 10^{4}$ and $7.6 \times 10^{5}$ years [9]. Relative to molecular hydrogen the minimum $\mathrm{HCN}$ abundance obtained in the clumps of the slow cycling models is $1.2 \times 10^{-8}$ at $3 \times 10^{6}$ years [25]. Prior to one million years of the clump phase the HCN abundance is never less than $1.0 \times 10^{-8}$ and is $\sim 10^{-7}$ at $t_{\min }$. Studies of N-chemistry in the rapid cycling models of B5 have not yet been performed.

Two possibilities can be inferred from the above. First, the cycling time in B5 may be longer than $5 \times 10^{6}$ years: continued accretion in the clump phase would eventually reproduce a low HCN abundance similar to that observed. The second possibility is that $x_{e}<10^{-3}$ in the preshock gas, this could be due to inefficient mixing or to significant recombination having occurred in the mass-loaded wind. The J-shocks of the slow cycling models with mixing have peak postshock temperatures in the range $3000-4000 \mathrm{~K}$ and produce copious amounts of HCN [25]. When the pre-shock $x_{e}$ is lower the shocks will be C-type, have significantly lower maximum post-shock temperatures $\sim 1000 \mathrm{~K}$, and may be expected to produce less HCN. Observations of other molecules such as $\mathrm{SO}, \mathrm{SO}_{2}$ and $\mathrm{H}_{2} \mathrm{~S}$ which are predicted to exhibit strong time dependence in their clump abundances [25] would help to further constrain the models.

A fundamental feature of the cycling models is the periodic return of mantle material to the gas by shock waves. In all the published models this was assumed to occur instantaneously with unit efficiency by an unspecified physical process. Although some calculations have recently been done in which the mantle removal efficiency is less than $100 \%$ (Nejad \& Williams; this volume), this aspect has not been treated consistently and is a critical weakness of the models. Shock speeds considered in the models are typically in the range $10-20 \mathrm{~km} \mathrm{~s}^{-1}$. In the slow cycling models, where the shocks are J-type, the pre-shock $\mathrm{H}$ nucleon density, $n_{o}$, is $400 \mathrm{~cm}^{-3}$. Calculations show that under these conditions no mantle removal is possible [20]. If $x_{e}$ is $10^{-3}$ or less the shocks will be Ctype, however, calculations show that mantle removal by grain-neutral sputtering will be inefficient for the model parameters considered [14]. Betatron acceleration, grain-grain collisions, or ion-grain collisions, could be important for mantle removal in the slow models but these processes have not been investigated quantitatively. Mantle removal in wind-clump interfaces has been suggested (e.g. [11]) but this remains speculative at present. It therefore appears that mantle removal in the slow cycling models is an inefficient process: these models should eventually deplete the heavy component of the gas phase in a similar manner as static ones [4]. 
In the C-type shocks of the rapid cycling models, the preshock ionisation and density are about $10^{-7}$ and $10^{4} \mathrm{~cm}^{-3}$ respectively. For the expected magnetic field strengths substantial mantle removal can occur via grain-neutral sputtering for shock speeds in excess of about $18 \mathrm{~km} \mathrm{~s}^{-1}$ [14]. The rapid cycling models appears to be the only ones in which mantles can be efficiently removed and more work is required to determine whether this process is viable in the slow models.

\section{Conclusions}

Theoretical models of star-forming regions must attempt to include a realistic treatment of the associated dynamical processes. Models of molecular clouds developed in this manner can plausibly provide natural answers to problems connected with more conventional, static ones e.g. mantle removal, $\mathrm{C} / \mathrm{CO}$ ratio, existence of 'young' chemistries as opposed to steady-state ones. Several theoretical problems do exist with the current models. In particular, a more consistent treatment of shock structure and the physics of mantle removal are required. Multi-molecular studies of particular star-forming regions are needed, especially of species which may act as diagnostics of the dynamics.

\section{References}

1. Bachiller, R., Menten, K. \& Rio-Alvarez, S. 1990, Astron. Astrophys., 236, 461.

2. Benson, P.J. \& Myers, P.C. 1989, Ap. J. Suppl., 71, 89.

3. Brown, P.D. \& Charnley, S.B. 1989, unpublished.

4. Brown, P.D. \& Charnley, S.B. 1990, M.N.R.A.S., 244, 432.

5. Cernicharo, J. 1990, in Physics of Star Formation and Early Stellar Evolution, NATO Advanced Study Institute, Crete, 1990.

6. Charnley, S.B., Dyson, J.E., Hartquist, T.W. \& Williams, D.A., 1988, M.N.R.A.S., 231, 269.

7. Charnley, S.B., Dyson, J.E., Hartquist, T.W. \& Williams, D.A., 1988, M.N.R.A.S., 235, 1257.

8. Charnley, S.B., Dyson, J.E., Hartquist, T.W. \& Williams, D.A., 1990, M.N.R.A.S., 243, 405.

9. Charnley, S.B., Whittet, D.C.B. \& Williams, D.A. \& 1990, M.N.R.A.S., 245, 161.

10. Charnley, S.B., Whittet, D.C.B. \& Williams, D.A. 1990, in Molecular Clouds, Eds. R.A. James \& T.J. Millar, Cambridge University Press, p321.

11. Charnley, S.B., \& Williams, D.A. 1990, in Molecular Astrophysics,

Ed. T.W. Hartquist, Cambridge University Press, p313.

12. d'Hendecourt, L.B., Allamandola, L.J. \& Greenberg, J.M., 1985, Astron. Astrophys., 152, 130.

13. Draine, B.T. 1980, Ap. J., 241, 1021.

14. Draine, B.T., Roberge, W.G. \& Dalgarno, A. 1983, Ap. J., 264, 485.

15. Fuller, G.A., et al. 1991, Ap. J., 376, 135.

16. Goldsmith, P.F., Langer, W.D. \& Wilson, R.W. 1986, Ap. J., 303, L11.

17. Goldsmith, P.F., Margulis, M., Snell, R.L., \& Fukui, Y. 1991, preprint.

18. Hartquist, T.W. \& Dyson, J.E. 1988, Astrophys. Space. Sci., 144,615.

19. Herbst, E. \& Leung, C.M. 1989, Ap. J. Suppl., 69, 271.

20. Hollenbach, D.J. \& McKee, C.F. 1979, Ap. J. Suppl., 41, 555.

21. Leung, C.M., Herbst, E. \& Huebner, W.F. 1984, Ap. J. Suppl., 56, 231.

22. Loren, R.B. \& Wooten, A. 1986, Ap. J., 306, 142.

23. Millar, T.J. 1990, in Molecular Astrophysics, Ed. T.W. Hartquist, Cambridge University Press, p115.

24. Mitchell, G.F. 1983, M.N.R.A.S., 205, 765.

25. Nejad, L.A.M., Williams, D.A. \& Charnley, S.B. 1990, M.N.R.A.S., 246, 183.

26. Norman. C. \& Silk, J. 1980, Ap. J., 251, 533.

27. Olano, C.A., Walmsley, C.M. \& Wilson, T.L. 1988, Astron. Astrophys., 196, 194.

28. Pineau des Forêts, Flower, D., Hartquist, T.W. \& Millar, T.J. 1987, M.N.R.A.S., 227, 993.

29. Tielens, A.G.G.M. \& Allamandola, L.J. 1987, in Physical Processes in Interstellar Clouds, Eds. G.E. Morfill \& M. Scholer., Kluwer, p.333.

30. Williams, D.A. \& Hartquist, T.W. 1984, M.N.R.A.S., 210, 141.

31. Williams, D.A. \& Hartquist, T.W. 1991, M.N.R.A.S., 251, 351. 


\section{QUESTIONS AND ANSWERS}

J.M.C.Rawlings: I would just like to point out that cosmic-ray induced photodissociation reactions (not included in the cyclic models of Nejad et al.) can have an important effect on the gas-phase chemistry (and consequently the initial mantle composition) in dynamical models in which freeze-out onto grains is significant. Abundance of saturated molecules (eg. $\mathrm{NH}_{3}, \mathrm{H}_{2} \mathrm{O}$ ) can be suppressed by over an order of magnitude. This is to be expected as the timescale for clump collapse, freeze-out and photodissociation are similar.

S.B.Charnley: It may be interesting to study cyclic models incorporating this process. The abundance of HCN in the slow models is 10 times that observed by Fuller et al., and is probably due to the reaction of $\mathrm{CN}$ with $\mathrm{H}_{2}$ in the postshock gas. 